EXTENDED REPORT

\title{
Proteomic analysis of secreted proteins in early rheumatoid arthritis: anti-citrulline autoreactivity is associated with up regulation of proinflammatory cytokines
}

\author{
Wolfgang Hueber, Beren H Tomooka, Xiaoyan Zhao, Brian A Kidd, Jan W Drijfhout, James F Fries, \\ Walther J van Venrooij, Allan L Metzger, Mark C Genovese, William H Robinson
}

See end of article for authors' affiliations

Correspondence to: Dr W Hueber, Division of Immunology and Rheumatology, Department of Medicine, Stanford University School of Medicine, Palo Alto VA Health Care System, MC $154 \mathrm{R}, 3801$ Miranda Avenue, Palo Alto, CA 94304, USA;

whueber@stanford.edu

Accepted 19 July 2006

Published Online First

10 August 2006

\begin{abstract}
Objectives: To identify peripheral blood autoantibody and cytokine profiles that characterise clinically relevant subgroups of patients with early rheumatoid arthritis using arthritis antigen microarrays and a multiplex cytokine assay.

Methods: Serum samples from 56 patients with a diagnosis of rheumatoid arthritis of $<6$ months' duration were tested. Cytokine profiles were also determined in samples from patients with psoriatic arthritis (PsA) and ankylosing spondylitis $(n=21)$, and from healthy individuals $(n=19)$. Data were analysed using KruskalWallis test with Dunn's adjustment for multiple comparisons, linear correlation tests, significance analysis of microarrays (SAM) and hierarchical clustering software.

Results: Distinct antibody profiles were associated with subgroups of patients who exhibited high serum levels of tumour necrosis factor (TNF) $\alpha$, interleukin (IL) 1 $\beta$, IL6, IL13, IL15 and granulocyte macrophage colonystimulating factor. Significantly increased autoantibody reactivity against citrullinated epitopes was observed in patients within the cytokine "high" subgroup. Increased levels of TNF $\alpha$, IL1 $\alpha$, ILI 2p40 and IL13, and the chemokines eotaxin/CCL1 1, monocyte chemoattractant protein-1 and interferon-inducible protein 10, were present in early rheumatoid arthritis as compared with controls $(p<0.001)$. Chemokines showed some of the most impressive differences. Only IL8/CXCL8 concentrations were higher in patients with PsA/ankylosing spondylitis $(p=0.02)$.

Conclusions: Increased blood levels of proinflammatory cytokines are associated with autoantibody targeting of citrullinated antigens and surrogate markers of disease activity in patients with early rheumatoid arthritis. Proteomic analysis of serum autoantibodies, cytokines and chemokines enables stratification of patients with early rheumatoid arthritis into molecular subgroups.
\end{abstract}

$\mathrm{R}$ heumatoid arthritis is an autoimmune disease that involves multiple molecules and pathways. Autoantibodies and cytokines represent classes of immune cellsecreted proteins postulated to have a variety of roles in rheumatoid arthritis, from regulating the initiation and perpetuation of chronic inflammatory responses to joint destruction. ${ }^{1-3}$ However, the precise mechanisms leading to the expression of autoantibodies and cytokines in early rheumatoid arthritis are not completely understood.

Although only scant evidence exists that autoantibodies are directly pathogenic in rheumatoid arthritis, they represent important markers for diagnosis and classification of rheumatoid arthritis. ${ }^{2}$ By contrast, autoantibodies have been observed infrequently in other types of arthritis. ${ }^{4}$ Proinflammatory cytokines such as tumour necrosis factor (TNF) $\alpha$ and interleukin (IL) l probably play important parts in regulating immune activation, driving the inflammatory process and promoting joint destruction in a variety of inflammatory joint diseases. ${ }^{5}$ Chemokines are chemotactic cytokines produced by fibroblast-like synoviocytes, cells of the innate immune system and other immunoregulatory cells, and there is solid evidence that, among their many roles, they are important potentiators of autoimmune arthritis. ${ }^{4}$ As expression of cytokines and chemokines in synovial tissue occurs early in the course of rheumatoid arthritis, ${ }^{78}$ they are under evaluation as biomarkers in early rheumatoid arthritis. The advent of proteomics technologies has enabled large-scale analysis of proteins to identify biomarkers that delineate disease subtypes of rheumatoid arthritis, and to gain insights into the mechanisms underlying these subtypes. We recently developed and applied antigen microarrays for the diagnosis and classification of rheumatoid arthritis and early rheumatoid arthritis.. ${ }^{10}$ We described 1536-feature arthritis antigen arrays containing 225 peptides and proteins representing candidate autoantigens in rheumatoid arthritis. ${ }^{9}$ Antigens included a wide variety of native and in vitro citrullinated proteins and peptides, which were robotically printed to the surface of microscope slides, where the binding of serum autoantibodies was detected. ${ }^{911}$

In this paper, we describe a multiplex analysis of serum cytokines using an optimised cytokine bead assay, and integration of these datasets with previously determined antigen array-derived autoantibody signatures. ${ }^{9}$ We tested the following hypotheses: (1) cytokines and chemokines derived from subsets of immunoregulatory cells are selectively upregulated in early rheumatoid arthritis; and (2) classes of cytokines are associated with distinct patterns of autoantibody reactivity. Our results provide new insights into associations of anticitrulline autoantibody responses with production of proinflammatory cytokines, highlight the potential of autoantibodies

Abbreviations: ARAMIS, Arthritis, Rheumatism, and Aging Medical Information System; CCP2, cyclic citrullinated peptide 2; FDR, false discovery rate; GM-CSF, granulocyte macrophage colony-stimulating factor; IFN, interferon; IP-10/CXCL10, interferon-inducible protein 10; MCP-1/CCL2, monocyte chemoattractant protein-1; PsA, psoriatic arthritis; SAM, significance analysis of microarrays; TNF, tumour necrosis factor 
and cytokines as biomarkers, and suggest a role for chemokines as additional biomarkers in early rheumatoid arthritis.

\section{PATIENTS AND METHODS Patients and sera}

All rheumatoid arthritis and control serum samples were obtained under Stanford University Institutional Review Board approved protocols and with informed consent. Samples from patients with ankylosing spondylitis and psoriatic arthritis $(\mathrm{n}=21)$, and from healthy individuals $(\mathrm{n}=19)$, were provided by a clinical reference laboratory (RDL, Los Angeles, California, USA). Owing to limitations in the number of arrays run in individual experiments, the Arthritis, Rheumatism, and Aging Medical Information System (ARAMIS) cohort samples studied comprised 56 randomly selected serum samples from 793 patients in the ARAMIS early rheumatoid arthritis inception cohort, ${ }^{12}$ collected from patients with a clinical diagnosis of rheumatoid arthritis (according to the revised American College of Rheumatology

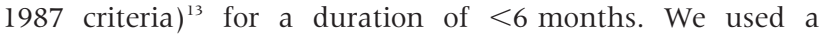
randomisation algorithm for selection of 56 serum samples from the ARAMIS sample bank. The baseline characteristics of this subgroup of patients with early rheumatoid arthritis were assessed and found comparable with those of the whole cohort of patients (table $1^{9}$ ), and their autoantibody responses had been previously characterised by antigen microarray assays. ${ }^{9}$

\section{Cytokine assay}

The human 22-cytokine Beadlyte kit (Upstate, Charlottesville, Virginia, USA) and the Luminex XMAP 100IS platform (Luminex, Austin, Texas, USA) were used according to the manufacturers' protocols, except for using $50 \%$ of the recommended serum and buffer volumes. For all experiments reported in this paper, unless stated otherwise, an additional blocking reagent optimised for sandwich immunoassays (HeteroBlock, Omega Biologicals, Bozeman, Montana, USA) was added to the serum sample buffer to achieve $3 \mu \mathrm{g} / \mathrm{ml}$ final concentration. Immunodepletion was performed by incubation of $100 \mu \mathrm{l}$ of serum with $25 \mu \mathrm{l}$ of protein L-sepharose beads (Pierce Biotechnologies, Rockford, Illinois, USA) for $30 \mathrm{~min}$ at $4^{\circ} \mathrm{C}$, followed by 30 -s centrifugation at $14 \mathrm{k}$ revolutions per minute and removal of the supernatant for cytokine analysis. Calibration controls and recombinant standards were used as specified by the manufacturer. Linear correlation coefficients and Mann-Whitney $U$ test statistics were calculated using InStat software. Cytokine concentrations were plotted, and p values calculated by Kruskal-Wallis tests with Dunn's multiple comparisons using Prism software.

\section{Microarrays}

Protocols for array production and data analysis were presented in prior work ${ }^{914}$ and are available online (www.stanford.edu/ group/robinsonlab). Previously generated antigen array datasets ${ }^{9}$ were integrated with newly generated cytokine array datasets for the analysis of associations between autoantibody profiles and cytokine profiles. Arrays were scanned using the GenePix4000 Scanner (Molecular Devices Corporation, Union City, California, USA). Median pixel intensities of features and background were determined using GenePix Pro V.3 software.

\section{Array data analysis}

Median net digital fluorescence units represent median values from 4-8 identical features on each array, and were normalised to the median intensity of 12-20 anti-immunoglobulin (Ig)M features. SAM ${ }^{15}$ identified antigens with statistical differences in array reactivity between subgroups of early rheumatoid arthritis, stratified on the basis of cytokine levels (fig 1).
Normalised median array values were mathematically adjusted and input into SAM, and results were selected based on false discovery rates (FDRs) and numerator thresholds to identify differentially targeted antigens that exhibited the greatest fold change in reactivities: fig $1 \mathrm{~A}, \mathrm{FDR}<0.08$ and a numerator threshold of 1.3; fig 1B, FDR $<0.14$ and a numerator threshold of 1.9; fig $1 \mathrm{C}, \mathrm{FDR}<0.41$ and a numerator threshold of 1.9; fig $1 D, F D R<0.11$ and a numerator threshold of 1.2; fig $1 \mathrm{E}$, FDR $<0.21$ and a numerator threshold of 1.9; and fig $1 \mathrm{~F}$, FDR $<0.22$ and a numerator threshold of 1.5.

SAM results were arranged as per relationships using Cluster software, and Cluster results displayed using TreeView software. $^{15}$ The CCP2 ELISA kits (Immunoscan RA Mark 2, Eurodiagnostica, Malmoe, Sweden) were used in accordance with the instructions of the manufacturer.

Determination of the shared epitope status and other parameters for the ARAMIS cohort have been described elsewhere. ${ }^{13}$

\section{RESULTS}

Broad up regulation of diverse serum cytokines and chemokines in early rheumatoid arthritis

We measured cytokine concentrations in a cohort of patients with early rheumatoid arthritis and controls, using an optimised 22-plex cytokine assay (see supplementary fig A at http://ard.bmjjournals.com/supplemental). In fig 2, the complete dataset of measurements derived from the early rheumatoid arthritis samples is represented as a hierarchical cluster heatmap for easy visualisation of cytokine signatures in individual patients. Strikingly, samples with detectable cytokines often had increased levels of several cytokines, including both the classical $\mathrm{T}$ helper (Th) 1 (interferon (IFN) $\gamma$ and IL12) and Th2 (IL10 and IL13) cytokines (fig 2A).

Two major umbrella clusters of patients emerged, a "high" cytokine/high inflammatory cluster and a "low" cytokine/low inflammatory cluster. The "high" cytokine/high inflammatory cluster was comprised of $21(37.5 \%)$ patients with early rheumatoid arthritis, characterised by a multi-cytokine signature. Figure 1B summarizes the marked differences in features between patients of these umbrella clusters. Of note, only laboratory (CCP ELISA, rheumatoid factor, eosinophil sedimentation rate, $\mathrm{C}$ reactive protein), and not the available clinical parameters of disease activity (HAQ scores, global assessment scores) differed markedly between patients of the two clusters.

Linear (Pearson's) correlation analysis showed strong correlations beween the Thl cytokines (eg, IFN $\gamma$ and IL12, R = 0.91) and Th2 cytokines (eg, IL4 and IL10, $\mathrm{R}=0.79$ ). Moderate to strong correlations were also observed between Th1 and Th2 cytokines-for example, IFN $\gamma$ and ILI0 $(\mathrm{R}=0.65)$, IFN $\gamma$ and IL6 $(R=0.65)$, and IL12p70 and ILl0 $(R=0.84)$. Anti-CCP2 ELISA positivity correlated moderately to strongly with positive rheumatoid factor results (Spearman's $\mathrm{R}=0.76,95 \%$ confidence interval $(\mathrm{CI}) 0.62$ to $0.86 ; \mathrm{p}<0.001)$. Correlations were also observed between CRP levels and IL6 concentrations $(\mathrm{R}=0.42 ; 95 \%$ CI 0.17 to $0.63 ; \mathrm{p}=0.001)$, and between CRP and MIP- $1 \alpha /$ CCL3 concentrations $(\mathrm{R}=0.38 ; 95 \% \mathrm{CI} 0.13$ to $0.59 ; \mathrm{p}=0.003)$.

Removing or blocking of heterophilic antibodies is essential for quantitative measurements of cytokines in rheumatoid factor seropositive sera

Bead-based multiplex cytokine assays have been validated by others, using both human blood (serum or plasma) and human peripheral blood mononuclear cell culture supernatants. ${ }^{16}{ }^{17}$ Multiplex assays were more reproducible and reliable than conventional ELISA-based measurements. ${ }^{17}{ }^{19-21}$ However, 


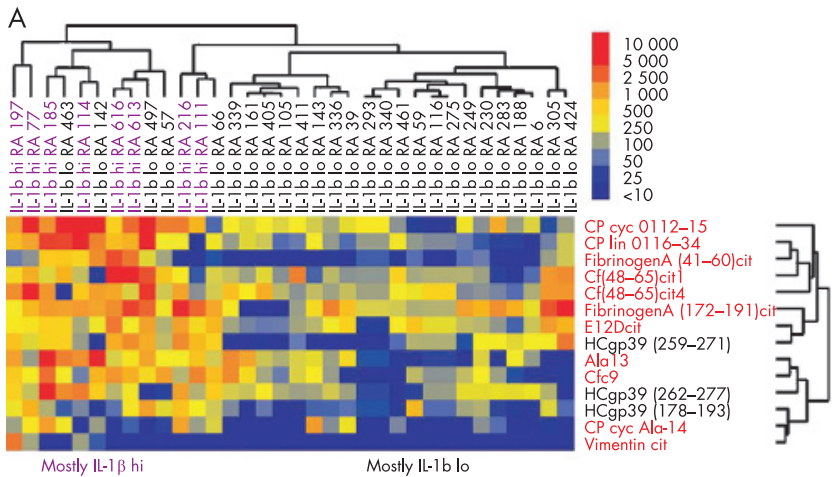

B
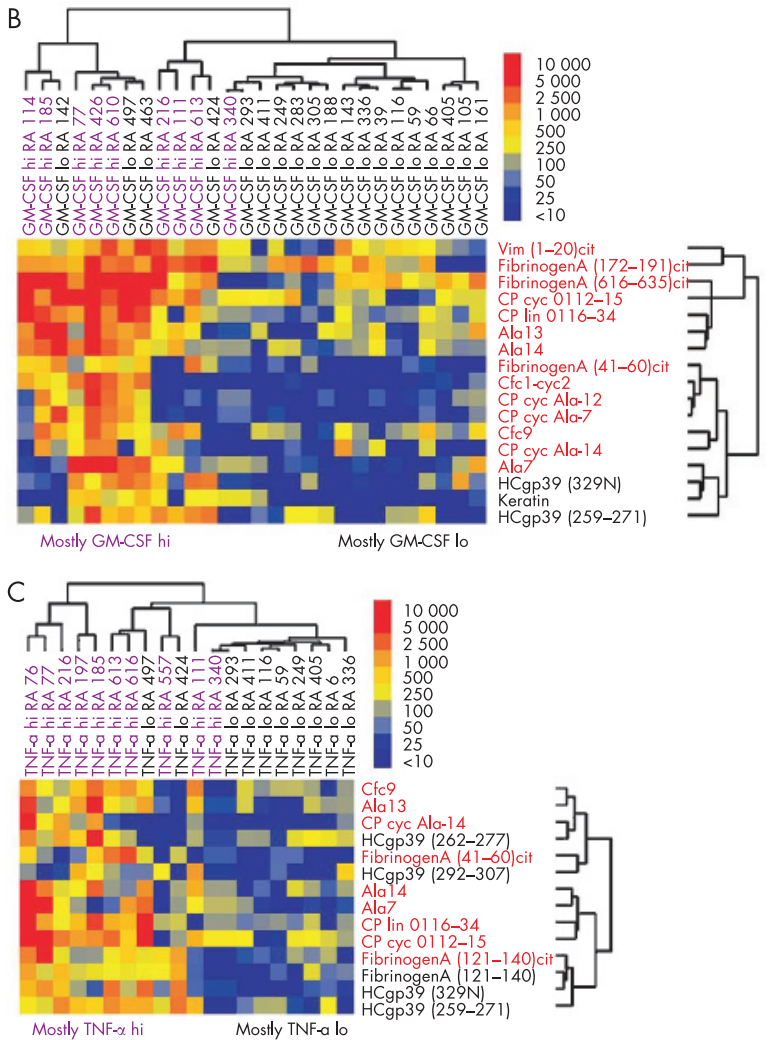

D
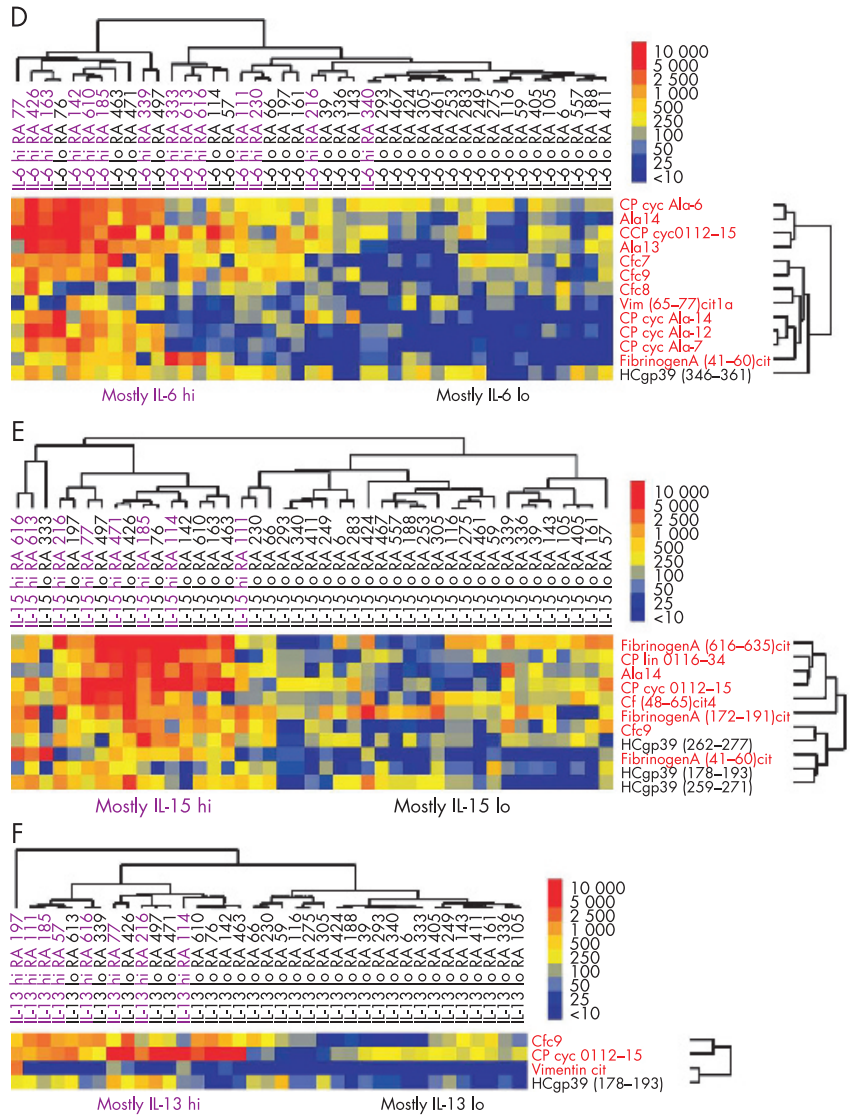

Figure 1 Differential targeting of citrullinated epitopes in subpopulations of patients with early rheumatoid arthritis with divergent serum cytokine levels. Autoantibody reactivity was determined by antigen arrays, and cytokine concentrations were determined by a bead-based multiplex cytokine assay in 56 early rheumatoid arthritis serum samples. Pairwise significance analysis of microarrays (SAM) was performed to identify antigen features with statistically significant differences in arthritis array reactivity that were associated with serum cytokine levels. Specific analyses include comparisons of female patients with rheumatoid arthritis who had increased or immeasurable serum levels of interleukin (IL)1 $\beta$ (A), granulocyte macrophage colony-stimulating factor (GMCSF) (B), tumour necrosis factor (TNF) \& (C), IL6 (D), IL15 (E) and IL13 (F). Hierarchical clustering was applied to arrange the patients and SAM-identified antigen features (dendrograms on the top and right, respectively). The labels below the cluster images indicate the general locations of the clustering of the sample type being compared. The labels to the right of the cluster images indicate the general locations of antigens, with the citrullinated antigens shown in red type.

concerns exist for both assays regarding the accuracy of measurements in blood or synovial fluid when interfering factors such as heterophilic antibodies are present. ${ }^{22}$ Heterophilic antibodies such as rheumatoid factors are defined as antibodies with multispecific activities directed against poorly defined antigens. ${ }^{22}$ Multiple studies have shown that blocking or depletion of heterophilic antibodies results in major reductions in read-out levels from cytokine immunoassays, suggesting that heterophilic antibodies including rheumatoid factor can result in false-positive signals in ELISAs and other immunoassays. 82425

In our preliminary experiments, we observed a striking association of increased serum concentrations of multiple cytokines with rheumatoid factor seropositivity (data not shown). To determine whether rheumatoid factor was causing false elevations in signal in our multiplex cytokine assay, we depleted the serum of immunoglobulins by incubation of $100 \mu \mathrm{l}$ of serum with $25 \mu \mathrm{l}$ of protein L-sepharose beads for $30 \mathrm{~min}$ at $4^{\circ} \mathrm{C}$. Depletion of immunoglobulins resulted in substantial reduction in signal in several rheumatoid factor seropositive samples (supplementary fig A). Measurements in rheumatoid factor seronegative samples were not affected (see supplementary fig $C$ in http://ard.bmjjournals.com/supplemental, sample 12; also data not shown). Importantly, a substantial fraction of rheumatoid factor seropositive samples showed very low or undetectable concentrations of several cytokines (fig 2A). 
A

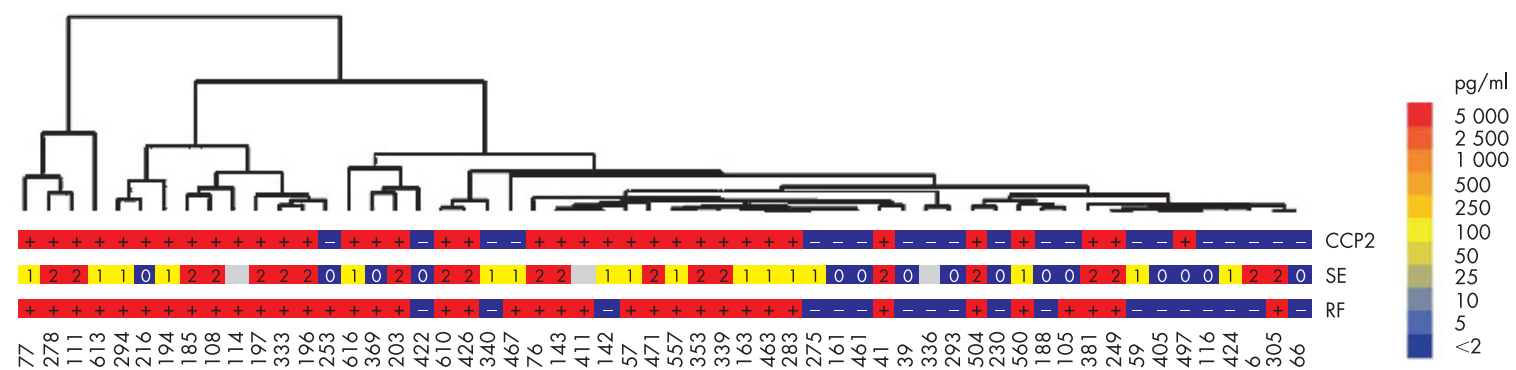

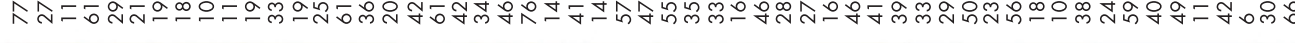

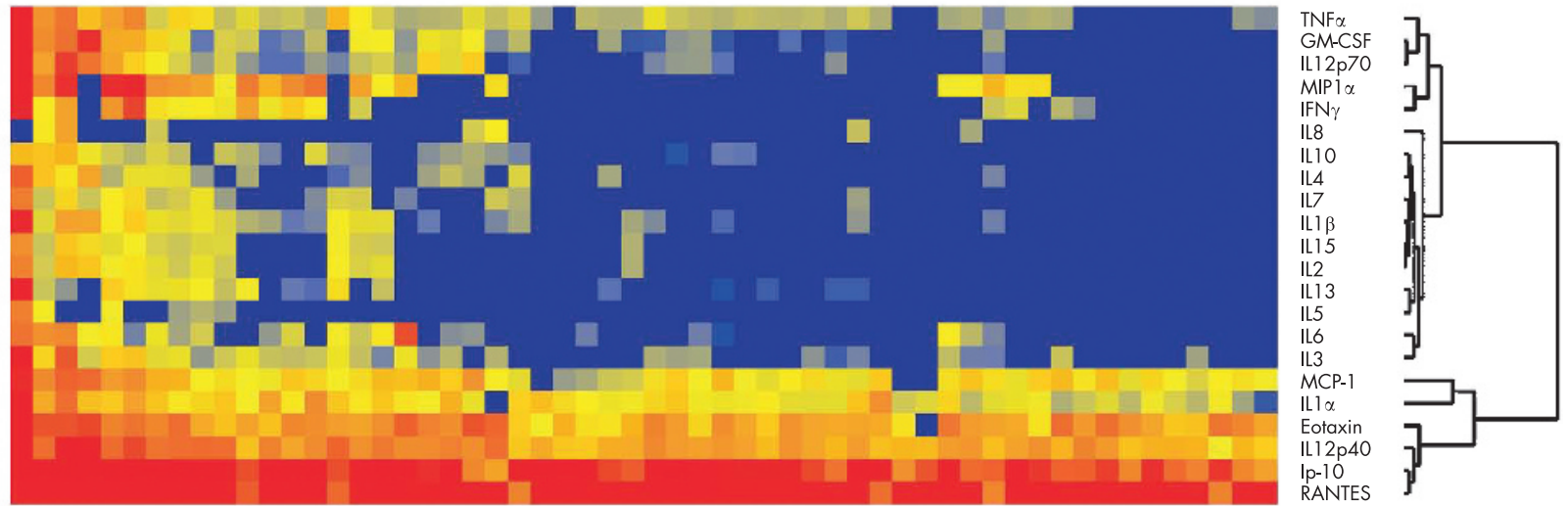

Cytokine "high" umbrella cluster

Cytokine "low" umbrella cluster

B

\begin{tabular}{llll}
\hline & $\begin{array}{l}\text { Cytokine high umbrella cluster } \\
(\mathrm{n}=21) ; \text { medians }\end{array}$ & $\begin{array}{l}\text { Cytokine low umbrella cluster } \\
(\mathrm{n}=35) ; \text { medians }\end{array}$ & $\begin{array}{l}\mathrm{p} \text { Value } \\
\text { (Mann-Whitney U test) }\end{array}$ \\
\hline Age & 54 & 53 & \\
CRP & $1.10 \mathrm{mg} / \mathrm{dl}$ & $0.33 \mathrm{mg} / \mathrm{dl}$ & 0.001 \\
ESR & $38.5 \mathrm{~mm} \mathrm{Hg}$ & $19 \mathrm{~mm} \mathrm{Hg}$ & 0.045 \\
RF titre & 285.0 & 32.1 & $<0.001$ \\
CCP titre & 472.2 & 28.0 & 0.024 \\
HAQ & 1.25 & 1.125 & $\mathrm{NS}$ \\
GLOBAL & 33 & 45 & $\mathrm{NS}$ \\
\hline
\end{tabular}

C

\begin{tabular}{lll}
\hline \multicolumn{3}{c}{ Linear correlation analysis } \\
\hline Cytokine & Anti-CCP2 and cytokine R; $(95 \%$ Cl) & p Value \\
\hline TNF $\alpha$ & $0.39(0.15$ to 0.59$)$ & 0.003 \\
IL10 & $0.38(0.13$ to 0.48$)$ & 0.004 \\
IL1 $\beta$ & $0.37(0.12$ to 0.58$)$ & 0.005 \\
IL 1 $\alpha$ & $0.36(0.10$ to 0.57$)$ & 0.007 \\
IL2 & $0.36(0.10$ to 0.57$)$ & 0.007 \\
IL4 & $0.35(0.10$ to 0.56$)$ & 0.008 \\
IL12p70 & $0.34(0.08$ to 0.56$)$ & 0.01 \\
IP10/CXCL10 & $0.32(0.06$ to 0.54$)$ & 0.02 \\
GM-CSF & $0.31(0.05$ to 0.53$)$ & 0.02 \\
IFN $\gamma$ & $0.28(0.014$ to 0.50$)$ & 0.04 \\
IL6 & $0.22(-0.05$ to 0.46$)$ & $\mathrm{NS}$ \\
IL13 & $0.22(-0.05$ to 0.46$)$ & $\mathrm{NS}$ \\
IL15 & $0.22(-0.05$ to 0.46$)$ & $\mathrm{NS}$
\end{tabular}

\section{Anti-CCP2 and RF titre: Spearman $\mathrm{R}=0.76 ; 95 \% \mathrm{Cl} 0.62$ to $0.86, \mathrm{p}<0.001$}

Figure 2 Blood cytokine profiles stratify patients with early rheumatoid arthritis. We applied a bead-based array using an optimised protocol to profile cytokines in rheumatoid arthritis serum samples. (A) Array results are displayed as a heat map after hierarchical clustering of all data points to visualise the spectrum of cytokine levels for each patient. Columns represent individual patients, labelled on the top. Red represents the highest cytokine values. For each patient, the number of copies of the shared epitope, rheumatoid factor status and CCP2 ELISA reactivity are indicated across the top of the panel. Rows representing individual cytokine levels are labelled on the right side of the panel. (B) Comparison between disease activity parameters of patients in the cytokine "high" and "low" umbrella clusters, respectively. (C) Linear correlation analysis was performed to determine correlations between anti-CCP2 and cyłokine concentrations. Correlation coefficients for selected individual pairs are displayed in descending order. 
Table 1 Baseline characteristics of the Arthritis, Rheumatism, and Aging Medical Information System patients analysed on arthritis arrays and with a multiplex cytokine assay $(n=56)^{*}$

\begin{tabular}{ll}
\hline Age, median (range), years & $53.5(19-78)$ \\
Female sex, n (\%) & $43(77)$ \\
RF seropositive, n (\%) & $38(68)$ \\
CRP median (range), mg/dl & $0.50(0.09-15.7)$ \\
Median (range) disability score & $1.125(0-2.375)$ \\
Median (range) educational level score & $12(8-17)$ \\
Treatment with conventional DMARD, n (\%) & $23(41)$ \\
Shared epitope present, no (\%) & $38(68)$
\end{tabular}

$\mathrm{CRP}, \mathrm{C}$-reactive protein; DMARD, disease-modifying anti-rheumatic drug $\mathrm{RF}$, rheumatoid factor

HeteroBlock is a reagent optimised to prevent rheumatoid factor from bridging capture and detection antibodies in sandwich immunoassays. Heteroblock was used previously to reduce non-specific binding of rheumatoid factor to primary and secondary antibodies in cytokine ELISAs. ${ }^{25}$ In our experiments, $3 \mu \mathrm{g} / \mathrm{ml}$ of HeteroBlock in serum diluent yielded effects comparable to immunoglobulin depletion by protein L-sepharose precipitation (see supplementary fig B in http://ard.bmjjournals.com/supplemental). Our observations are in line with recent experiments by de Jager et al, who showed that nearcomplete $(89 \%)$ depletion of plasma IgM rheumatoid factor by protein L-sepharose and additional blocking with 10\% rodent serum before bead-based multiplex cytokine analysis resulted in reduced non-specific binding and more accurate recovery rates of cytokines. ${ }^{18}$

Serum concentrations of the cytokines IL $1 \alpha, \operatorname{TNF} \alpha$, IL 12 p40 and IL 13 are increased in patients with early rheumatoid arthritis compared with controls

Comparisons of cytokine concentrations between early rheumatoid arthritis, PsA/ankylosing spondylitis and controls were performed using Kruskal-Wallis test, with post-test analysis by Dunn's multiple comparisons. Serum concentrations of the following cytokines were significantly increased in patients with early rheumatoid arthritis: ILl $\alpha(\mathrm{p}<0.001)$, TNF $\alpha$ $(\mathrm{p}<0.001)$ and IL12p40 $(\mathrm{p}<0.001$; fig $3 \mathrm{~A}, \mathrm{C}, \mathrm{D})$, and IL13 $(p=0.02$, data not shown). Significant differences were not observed for IL6, for which median concentrations did not differ beween PsA/ankylosing spondylitis and early rheumatoid arthritis (fig 3B).

Serum concentrations of the chemokines interferon-inducible protein 10 (IP-10/CXCL10), monocyte chemoattractant protein-1 (MCP-1/CCL2) and eotaxin/CCL1 1 were raised in patients with early rheumatoid arthritis compared with controls

Median serum concentrations of three chemokines were higher in early rheumatoid arthritis: IP-10/CXCL10 $(\mathrm{p}<0.001)$, eotaxin/CCL11 $(\mathrm{p}<0.001)$ and MCP-1/CCL2 ( $\mathrm{p}=0.001$; fig 3EG). IL-8/CXCL8 was the only cytokine with higher median concentrations in patients with PsA/ankylosing spondylitis compared with those with early rheumatoid arthritis $(\mathrm{p}=0.02$; fig $3 \mathrm{H})$. Although included in the multiplex cytokine assay, we did not analyse data for the chemokine RANTES/ CCL5, because a large number of measurements were out of range of quantification due to saturation of the beads with this cytokine at the standard serum dilution of $1: 1$ (data not shown).

\section{Integration of autoantibody profiles with cytokine concentrations}

To integrate cytokine profiles with autoantibody profiles, we performed a pairwise SAM analysis of arthritis array results from patients stratified based on the presence of raised and low/undetectable serum concentrations of cytokines. In this cross-sectional dataset, we determined which cytokines were associated with distinct antibody profiles and surrogate markers of disease activity and severity (CRP and HAQ) in early rheumatoid arthritis. Cut-offs for sample classification into the high cytokine subgroup were defined as samples above the 75 th centile. Patients were stratified according to sex and rheumatoid factor. These analyses showed significantly increased autoantibody reactivity against citrullinated epitopes in patients in the high cytokine subgroup, and subset analysis of women alone showed even stronger correlations (fig $\mathrm{lA}-\mathrm{F}$ ). IL1 $\beta$ "low", IL6 "low" and IL15 "low" subgroups of patients exhibited lower reactivity against citrullinated epitopes (fig $\mathrm{IA}, \mathrm{D}, \mathrm{E}$ ), and a trend towards targeting certain native epitopes including collagen type II, heterogenous nuclear ribonucleoprotein $\mathrm{B}$ and heterogenous nuclear ribonucleoprotein peptides (data not shown).

Comparison of the two major clusters shown in fig $2 \mathrm{~A}$ showed significantly higher CRP $(p=0.001)$, ESR $(p=0.045)$, CCP $(p=0.024)$ and RF titres $(p=0.001)$ within the "high" cytokine group (fig $2 \mathrm{~B}$ ). This observation indicates that the multi-cytokine signature defining the "high" cytokine cluster represents an additional biomarker in patients with more active disease, as measured by standard laboratory markers of disease activity.

\section{DISCUSSION}

We describe the application of arthritis antigen microarrays and a bead-based cytokine assay to profile secreted immunoregulatory proteins, including autoantibodies and cytokines, in blood derived from patients with early rheumatoid arthritis. We identified proteomic patterns of differential antigen recognition and cytokine production that differentiated a high inflammatory from a low inflammatory subtype of early rheumatoid arthritis. Several citrullinated epitopes and a few native human cartilage gp39 peptides were preferentially targeted by autoantibodies in patients with high serum levels of the proinflammatory cytokines TNF $\alpha$, IL1 $\beta$, IL6, IL15 and granulocyte macrophage colony-stimulating factor (GM-CSF), as well as IL13, and these patients possessed features predictive for the development of more severe arthritis. Moreover, our results suggest an important role for the downstream amplifiers of inflammatory responses in early rheumatoid arthritis, as three major chemokines were upregulated in patients with rheumatoid arthritis over controls, namely IP-10/CXCL10, a ligand of CXCR3 associated with Th1type reactions, ${ }^{26}$ eotaxin/CCL11, a ligand of CCR3 associated with Th2-type reactions, ${ }^{27}$ and MCP-1/CCL2

Little is known about the association of serum autoantibodies with serum cytokines and chemokines. In a study involving a very small number of patients with rheumatoid arthritis, a generalised up regulation of serum cytokine concentrations over that of controls was observed. ${ }^{28}$ Another recent study on early undifferentiated arthritis showed correlations between raised concentrations of multiple cytokines with clinical subtypes and anti-CCP ELISA antibody responses in early rheumatoid arthritis. ${ }^{28}$ In contrast to Hitchon et al, ${ }^{29}$ we did not observe statistically significant increases in the classical Th2 cytokine IL4 in rheumatoid arthritis serum compared with controls. This discrepancy might be due to our more aggressive sample treatment with HeteroBlock, which corrected for false elevations of several cytokines in rheumatoid factor seropositive samples (fig 2A, see supplementary fig B in http://ard.bmjjournals.com/supplemental; also data not shown). Moreover, we 

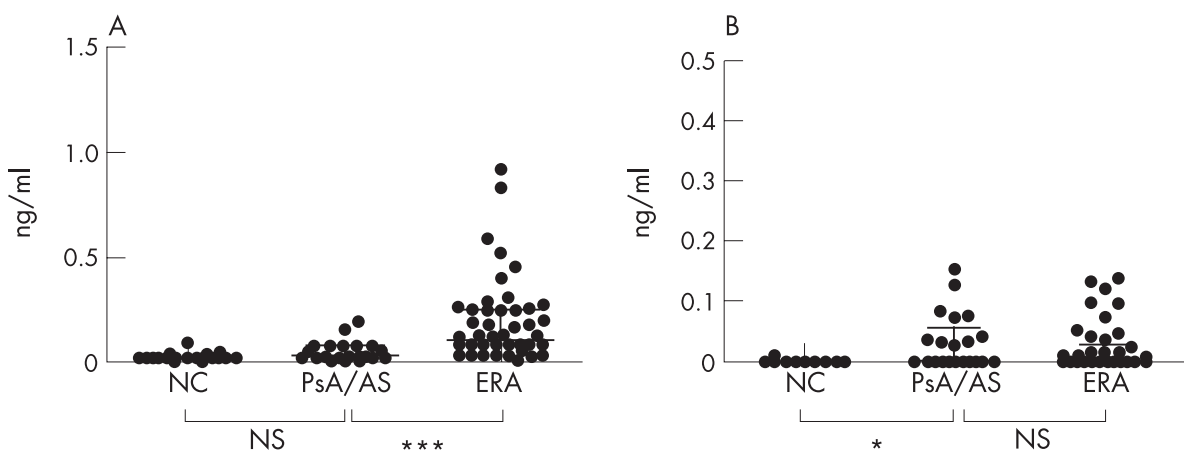

Figure 3 Comparison of cytokine concentrations in healthy individuals, patients with psoriatic arthritis $(\mathrm{Ps} A)$ and ankylosing spondylitis, and early rheumatoid arthritis. Serum samples from patients with early rheumatoid arthritis $(n=56), \operatorname{PsA}$ and ankylosing spondylitis $(n=21)$, and from healthy subjects $(n=19)$ were analysed by multiplex cytokine assay. Representative results are shown for (A) interleukin (IL) $1 \alpha$, (B) IL6, (C) tumour necrosis factor (TNF) $\alpha$, (D) IL12p40, (E) interferon-inducible protein 10 (IP-10/CXCL10), (F) eotaxin/CCL1 1, (g) monocyte chemoattractant protein (MCP-1/ CCL2) and (h) IL8/CXCL8. Horizontal bars
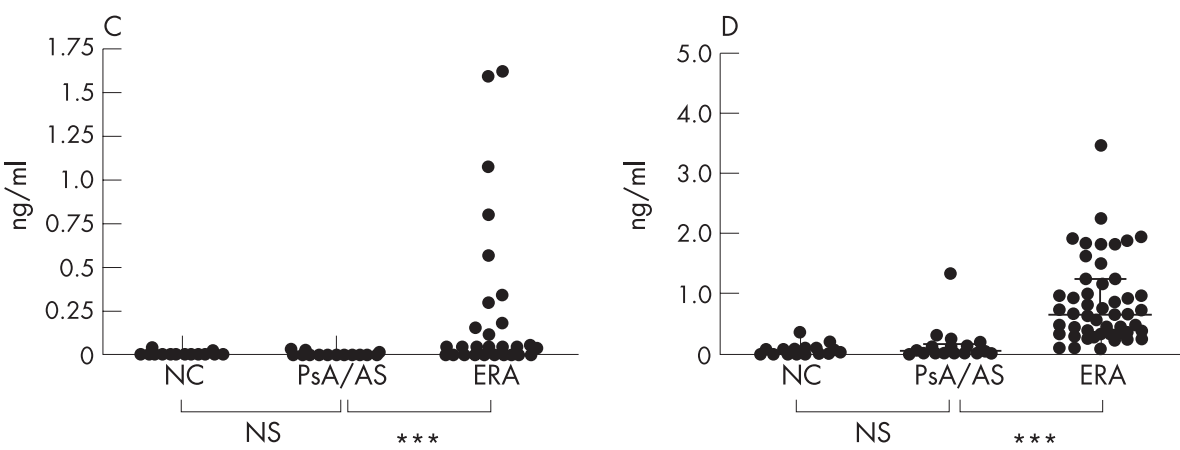
represent medians with centiles for each column.
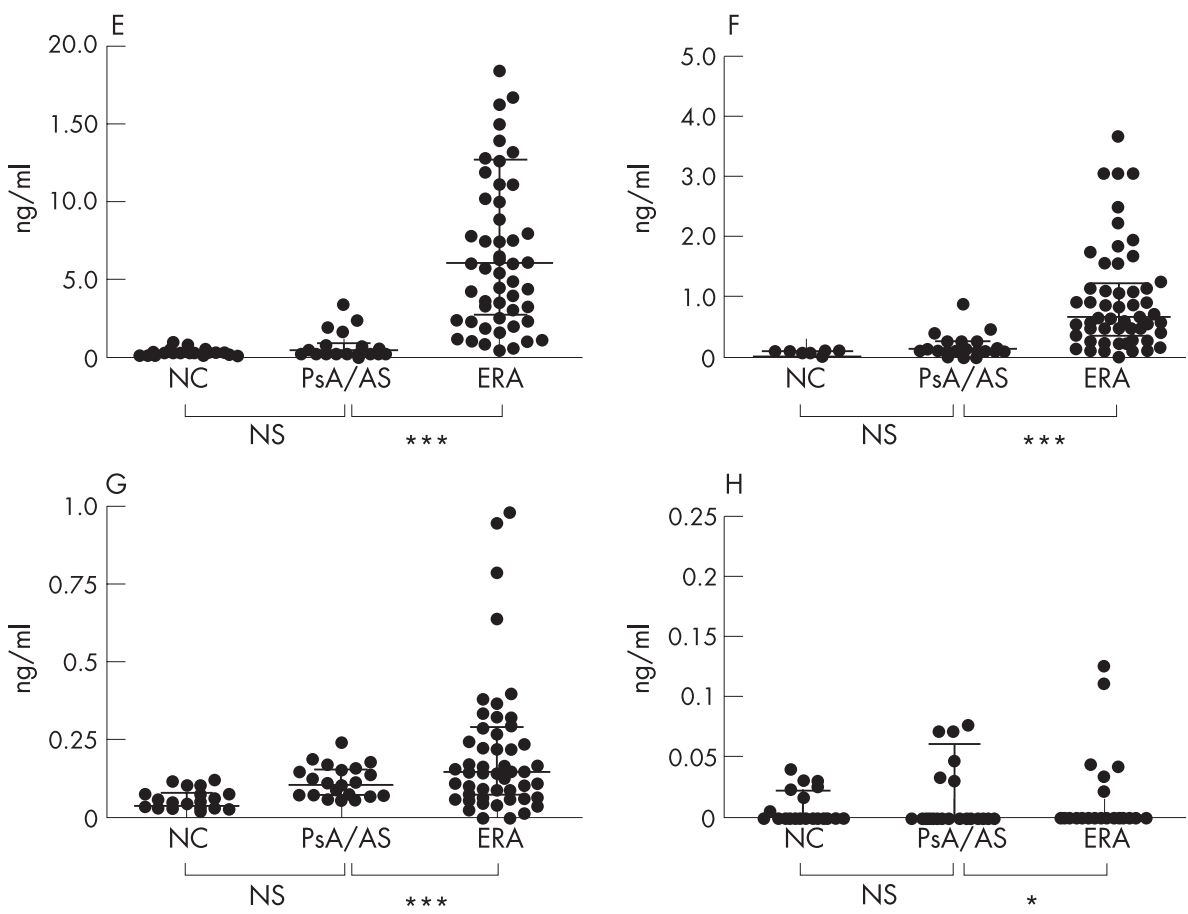

observed raised serum levels of both classical Thl (IFN $\gamma$ and IL12) and Th2 (IL10 and IL13) cytokines in about one third of patients with early rheumatoid arthritis (clustering to the left of the heatmap in fig 2A). Our results are consistent with the observation that dichotomous Th1 and Th2 T cells, although delineated in mice, have not been readily identified in humans. ${ }^{30}$

As patients in the ARAMIS cohort were not treated with anticytokine or other biological treatments at the time blood samples were obtained, serum concentrations in these samples may be more reflective of systemic levels of proinflammatory cytokines than in patients treated with cytokine-antagonising biological agents. ${ }^{31}$ Serum cytokine levels may reflect the level of immune cell activation in involved joints ${ }^{3}$ or lymphoid tissues. Our findings suggest that generation of autoantibodies against citrullinated epitopes and other antigens is linked predominantly to the production of high levels of proinflammatory cytokines by activated $\mathrm{T}$ cells, macrophages and other cells in rheumatoid arthritis. For instance, we observed associations of GM-CSF levels with anti-citrulline reactivity (fig 1B). As GM-CSF has been implicated in up regulation of class II MHC on human monocytes, ${ }^{32}{ }^{33}$ it is plausible that an immunological link between GM-CSF production, autoantigen presentation and induction of autoantibody production exists.

Recent work has suggested that chemokines have a prominent role in rheumatoid arthritis, and hence these modulators were 
proposed, together with their respective receptors, as targets for next generation therapeutics. ${ }^{64-36}$ Remarkably, our findings show that three chemokines, IP-10/CXCL10, eotaxin/CCL11 and MCP-1/CCL2, were strikingly increased in early rheumatoid arthritis serum compared with control serum (fig 3E-G). Chemokines such as IP-10/CXCL10 may have pivotal roles in recruiting activated $\mathrm{T}$ cells to sites of inflammation, and were proposed as key mediators of $\mathrm{T}$ cell polarisation in animal models of Thl-type autoimmunity. ${ }^{37} 38$ Other chemokines, including IL8/ CXCL8 and MCP-1/CCL2, ${ }^{39}{ }^{40}$ and GM-CSF ${ }^{41}$ are linked to TNF $\alpha$ via positive feedback loops, and are additive or even synergistic in their pathophysiological effects. Although expression of macrophage-associated chemokines has been described in the synovium of patients with early rheumatoid arthritis, their precise role in early synovitis, and their potential role as biomarkers in early rheumatoid arthritis, remains ill-defined. ${ }^{78}$ Longitudinal studies in a larger cohort of early rheumatoid arthritis including preclinical samples are under way to further elucidate the evolution of cytokine, chemokine and autoantibody signatures in early rheumatoid arthritis.

Multiplex assays enable cost-effective simultaneous measurements of serum cytokines, chemokines and autoantibodies. As robust assays become available for use in clinical laboratories, we expect that proteomic analyses will become a mainstay in the evaluation of patients with rheumatoid arthritis and other autoimmune diseases for assessing prognosis, guiding treatment and monitoring response to treatment.

\section{ACKNOWLEDGEMENTS}

We thank D Mathis, C Benoist and P Monach (Harvard Medical School, Boston, Massachusetts, USA), G Panayi (Guy's Hospital, London, UK), G Pruijn (Radboud University Nijmegen, Nijmegen, The Netherlands), G Steiner and JS Smolen (Vienna Medical School, Vienna, Austria) and $S$ Muller (Institut de Biologie Moleculaire et Cellulaire, Strasbourg, France) for providing antigens and sera. We thank $\mathrm{H}$ Neuman de Vegvar and R Tibshirani (Stanford) for insightful discussions, and the members of the Robinson and Utz laboratories for scientific input.

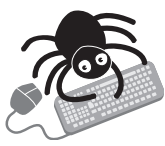

For supplementary figures see at http://ard. bmijournals.com/supplemental

\section{Authors' affiliations \\ Wolfgang Hueber, Beren H Tomooka, Xiaoyan Zhao, Brian A Kidd, James F Fries, Mark C Genovese, William H Robinson, Division of} Immunology and Rheumatology, Stanford University School of Medicine, Stanford, California, USA

Wolfgang Hueber, Beren H Tomooka, Xiaoyan Zhao, Brian A Kidd, William H Robinson, GRECC, Veterans Affairs Palo Alto Health Care System, Palo Alto, California, USA

Jan W Driifhout, Leiden University Medical Center, Leiden, The Netherlands

Walther J van Venrooij, Radboud University Niimegen, Niimegen, The Netherlands

Allan L Metzger, Rheumatology Diagnostics Laboratory, Los Angeles,

California, USA

Funding: This work was funded by a Weiland Family Fellowship Award to WH; National Institutes of Health (NIH) K08 AR02133, Arthritis Foundation Chapter Grants and an Investigator Award, NIH NHLBI contract N01 HV 28183, and Veterans Affairs Health Care System funding to WHR; and ARAMIS NIH AR043584 to JFF.

Competing interests: None.

All rheumatoid arthritis and control serum samples were obtained under Stanford IRB (Stanford University Institutional Review Board, Stanford University, Stanford CA 94305) approved protocols and with informed consent.

\section{REFERENCES}

1 Ferrara JL. Cytokines and the regulation of tolerance. J Clin Invest 2000;105: 1043-4

2 Firestein GS. Evolving concepts of rheumatoid arthritis. Nat Immunol 2003;432:356-61.

3 O'Shea JJ, Ma A, Lipsky P. Cytokines and autoimmunity. Nat Rev Immunol 2002;2:37-45

4 Smolen JS, Steiner G. Rheumatoid arthritis is more than cytokines: autoimmunity and rheumatoid arthritis. Arthritis Rheum 2001;44:2218-20.

5 Feldmann M, Maini RN. Lasker Clinical Medical Research Award. TNF defined as a therapeutic target for rheumatoid arthritis and other autoimmune diseases. Nat Med 2003;9:1245-50.

6 Charo IF, Ransohoff RM. The many roles of chemokines and chemokine receptors in inflammation. N Engl J Med 2006;354:610-21.

7 Katrib A, Tak PP, Bertouch JV, Cuello C, McNeil HP, Smeets TJM, et al. Expression of chemokines and matrix metalloproteinases in early rheumatoid arthritis. Rheumatology 2001;40:988-94.

8 Raza K, Falciani F, Curnow SJ, Ross E, Lee C-Y, Akbar A, et al. Early rheumatoid arthritis is characterized by a distinct and transient synovial fluid cytokine profile of T cell and stromal cell origin. Arthritis Res Ther 2005;7:R784-95

9 Hueber W, Tomooka BH, Kidd BA, Lee BJ, Bruce B, Fries JF, et al. Antigen microarray analysis defines subtypes of rheumatoid arthritis. Arthritis Rheum 2005;52:2645-55.

10 Hueber W, Utz P, Robinson W. Autoantibodies in early arthritis: advances in diagnosis and prognostication. Clin Exp Rheumatol 2003;21:S59-64.

11 Robinson W, DiGennaro C, Hueber W, Haab B, Kamachi M, Dean E, et al. Autoantigen microarrays for multiplex characterization of autoantibody responses. Nat Med 2002;8:295-301.

12 Fries J, Wolfe F, Apple R, Erlich H, Bugawan T, Holmes T, et al. HLA-DRB 1 genotype associations in 793 white patients from a rheumatoid arthritis inception cohort: frequency, severity, and treatment bias. Arthritis Rheum 2002;46:2320-9.

13 Arnett FC, Edworthy SM, Bloch DA, McShane DY, Fries JF, Cooper NS, et al. The American Rheumatism Association 1987 revised criteria for the classification of rheumatoid arthritis. Arthritis Rheum 1998;31:315-24.

14 Robinson W, Fontoura P, Lee B, de Vegvar H, Tom J, Pedotti R, et al. Protein microarrays guide tolerizing DNA vaccine treatment of autoimmune encephalomyelitis. Nat Biotechnol 2003;21:1033-9.

15 Tibshirani R, Hastie T, Narashimhan B, Chu G. Multi-class diagnosis of cancers using shrunken centroids of gene expression. Proc Natl Acad Sci USA 2002;99:6567-72

16 Eisen MB, Spellman PT, Brown PO, Botstein D. Cluster analysis and display of genome-wide expression patterns. Proc Natl Acad Sci USA 1998;95:14863-8.

17 Carson RT, Vignali AA. Simultaneous quantification of 15 cytokines using a multiplexed flow cytometric assay. J Immunol Methods 1999;227:41-52.

18 de Jager W, Prakken BJ, Bijlsma JWJ, Kuis W, Rijkers GT. Improved multiplex immunoassay performance in human plasma and synovial fluid following removal of interfering heterophilic antibodies. J Immunol Methods 2005;300:124-35

19 Chen R, Lowe L, Wilson JD, Crowther E, Tzeggai K, Bishop JE, et al. Simultaneous quantification of six human cytokines in a single sample using microparticle-based flow cytometric technology. Clin Chem 1999:45:1693-4.

20 Cook EB, Stahl JL, Lowe L, Chen R, Morgan E, Wilson J, et al. Simultaneous measurement of six cytokines in a single sample of human tears using microparticle-based flow cytometry: allergics vs. non-allergics. J Immunol Methods 2001;254:109-18.

21 Kellar K, Kalwar RR, Dubois KA, Crouse D, Chafin WD, Kane B-E. Multiplexed fluorescent bead-based immunoassays for quantitation of human cytokines in serum and culture supernatants. Cytometry 2001;45:27-36.

22 Aziz N, Nishanian P, Mitsuyasu R, Detels R, Fahey JL. Variables that affect assays for plasma cytokines and soluble activation markers. Clin Diagn Lab Immunol 1999;6:89-95.

23 Kaplan IV, Levinson SS. When is a heterophile antibody not a heterophile antibody? When it is an antibody against a specific immunogen. Clin Chem 1999:45:616-18.

24 Mclnnes IB, Leung BP, Sturrock RD, Field M, Liew FY. Interleukin-15 mediates T cell-dependent regulation of tumor necrosis factor-alpha production in rheumatoid arthritis. Nat Med 1997;3:189-95.

25 Shah MH, Hackshaw KV, Caligiuri MA. A role for IL-15 in rheumatoid arthritis. Nat Med 1998;4:643.

26 Qin S, Rottman JB, Myers P, Kassam N, Weinblatt $M$, Loetscher $M$, et al. The chemokine receptors CXCR3 and CCR5 mark subsets of T cells associated with certain inflammatory reactions. J Clin Invest 1998;101:746-54.

27 Gerber BO, Zanni MP, Uguccioni M, Loetscher M, Mackay CR, Pichler WJ, et al. Functional expression of the eotaxin receptor CCR3 in T lymphocytes colocalizing with eosinophils. Curr Biol 1997;7:836-43.

28 Stabler T, Piette JC, Chevalier X, Marini-Portugal A, Kraus VB. Serum cytokine profiles in relapsing polychondritis suggest monocyte/macrophage activation. Arthritis Rheum 2004;50:3663-7.

29 Hitchon CA, Alex P, Erdile LB, Frank MB, Dozmorov I, Tang Y, et al. A distinct multicytokine profile is associated with anti-cyclical citrullinated peptide antibodies in patients with early untreated inflammatory arthritis. $J$ Rheumatol 2004;31:2336-46.

30 Abbas A, Murphy K, Sher A. Functional diversity of helper T lymphocytes. Nature 1996:383:787-93.

31 Cutolo M, Capellino S, Montagna P, Sulli A, Seriolo B, Villaggio B. Antiinflammatory effects of leflunomide in combination with methotrexate on co- 
culture of T lymphocytes and synovial macrophages from rheumatoid arthritis patients. Ann Rheum Dis 2006;65:728-35.

32 Alvaro-Gracia JM, Zvaifler NJ, Firestein GS. Cytokines in chronic inflammatory arthritis. IV. Granulocyte/macrophage colony-stimulating factor-mediated induction of class II MHC antigen on human monocytes: a possible role in rheumatoid arthritis, J Exp Med 1989;170:865-75

33 Hornell TM, Beresford GW, Bushley A, Boss JM, Mellins ED. Regulation of the class II MHC pathway in primary human monocytes by granulocyte-macrophage colony-stimulating factor. J Immunol 2003;171:2374-83.

34 Garcia-Vicuna R, Gomez-Gaviro MV, Dominguez-Luis MJ, Pec MK, GonzalezAlvaro I, Alvaro-Gracia JM, et al. CC and CXC chemokine receptors mediate migration proliferation, and matrix metalloproteinase production by fibroblast-like synoviocytes from rheumatoid arthritis patients. Arthritis Rheum 2004;50: 3866-77.

35 Johnson Z, Schwarz M, Power CA, Wells TN, Proudfoot AE. Multi-faceted strategies to combat disease by interference with the chemokine system. Trends Immunol 2005;26:268-74.
36 Smolen JS, Steiner G. Therapeutic strategies for rheumatoid arthritis. Nat Rev Drug Discov 2003;2:473-88.

37 Salomon I, Netzer N, Wildbaum G, Schif-Zuck S, Maor G, Karin N. Targeting the function of IFN-gamma-inducible protein 10 suppresses ongoing adjuvant arthritis. J Immunol 2002:169:2685-93.

38 Wildbaum G, Netzer N, Karin N. Plasmid DNA encoding IFN-gamma-inducible protein 10 redirects antigen-specific T cell polarization and suppresses experimental autoimmune encephalomyelitis. J Immunol 2002; 168:5885-92.

39 Feldmann M, Maini RN. Anti-TNF alpha therapy of rheumatoid arthritis: what have we learned? Ann Rev Immunol 2001;19:163-96.

40 Gong JH, Ratkay LG, Waterfield JD, Clark-Lewis I. An antagonist of monocyte chemoattractant protein-1 1 (MCP-1) inhibits arthritis in the MRL-Ipr mouse model. J Exp Med 1997;186:131-7.

41 Cook AD, Braine EL, Campbell IK, Rich MJ, Hamilton JA. Blockade of collageninduced arthritis post-onset by antibody to granulocyte-macrophage colonystimulating factor (GM-CSF): requirement for GM-CSF in the effector phase of disease. Arthritis Res $2001 ; 3: 293-8$

\section{BMJ Clinical Evidence-Call for contributors}

BMJ Clinical Evidence is a continuously updated evidence-based journal available worldwide on the internet which publishes commissioned systematic reviews. BMJ Clinical Evidence needs to recruit new contributors. Contributors are healthcare professionals or epidemiologists with experience in evidence-based medicine, with the ability to write in a concise and structured way and relevant clinical expertise.

Areas for which we are currently seeking contributors:

- Secondary prevention of ischaemic cardiac events

- Acute myocardial infarction

- MRSA (treatment)

- Bacterial conjunctivitis

However, we are always looking for contributors, so do not let this list discourage you.

Being a contributor involves:

- Selecting from a validated, screened search (performed by in-house Information Specialists) valid studies for inclusion.

- Documenting your decisions about which studies to include on an inclusion and exclusion form, which we will publish.

- Writing the text to a highly structured template (about 1500-3000 words), using evidence from the final studies chosen, within 8-10 weeks of receiving the literature search.

- Working with BMJ Clinical Evidence editors to ensure that the final text meets quality and style standards.

- Updating the text every 12 months using any new, sound evidence that becomes available. The BMJ Clinical Evidence in-house team will conduct the searches for contributors; your task is to filter out high quality studies and incorporate them into the existing text.

- To expand the review to include a new question about once every 12 months. In return, contributors will see their work published in a highly-rewarded peer-reviewed international medical journal. They also receive a small honorarium for their efforts.

If you would like to become a contributor for BMJ Clinical Evidence or require more information about what this involves please send your contact details and a copy of your CV, clearly stating the clinical area you are interested in, to CECommissioning@bmigroup.com.

\section{Call for peer reviewers}

BMJ Clinical Evidence also needs to recruit new peer reviewers specifically with an interest in the clinical areas stated above, and also others related to general practice. Peer reviewers are healthcare professionals or epidemiologists with experience in evidence-based medicine. As a peer reviewer you would be asked for your views on the clinical relevance, validity and accessibility of specific reviews within the journal, and their usefulness to the intended audience (international generalists and healthcare professionals, possibly with limited statistical knowledge). Reviews are usually 1500-3000 words in length and we would ask you to review between 2-5 systematic reviews per year. The peer review process takes place throughout the year, and our turnaround time for each review is 10-14 days. In return peer reviewers receive free access to BMJ Clinical Evidence for 3 months for each review.

If you are interested in becoming a peer reviewer for BMJ Clinical Evidence, please complete the peer review questionnaire at www.clinicalevidence.com/ceweb/contribute/peerreviewer.jsp 